\title{
Simulação numérica do comportamento térmico do reservatório do Rio Verde
}

\section{Numerical simulation of the temperature dynamics at Rio Verde Reservoir}

\author{
Danieli Mara Ferreira \\ Engenheira Ambiental. Mestranda do Programa de Pós-Graduação de Engenharia de Recursos Hídricos e Ambiental (PPGERHA) da Universidade Federal do \\ Paraná (UFPR) - Curitiba (PR), Brasil.

\section{Cynara Cunha} \\ D.Sc. Professora Adjunta do curso de Engenharia Ambiental da Universidade Federal do Paraná (UFPR) - Curitiba (PR), Brasil. Pesquisadora do Laboratório \\ de Estudos em Modelagem e Monitoramento Ambiental (LEMMA) da UFPR - Curitiba (PR), Brasil.
}

\section{Resumo}

Mudanças na temperatura da água e na dinâmica de estratificação térmica podem causar efeitos negativos sobre os processos físicos, químicos e biológicos de reservatórios. A variação da temperatura afeta a densidade da água e, como consequência, altera os processos de transporte. Um modelo unidimensional de transferência de calor é utilizado para simular a temperatura do reservatório do Rio Verde, localizado na região metropolitana de Curitiba, Estado do Paraná. Na solução da equação, usou-se o método de diferenças finitas. O modelo foi calibrado e validado por meio dos dados de dois meses: julho de 2009 e março de 2010. Os resultados mostram uma boa concordância entre os valores simulados e observados. Esses resultados ainda permitem avaliar as relações da profundidade da termoclina com a radiação solar e a velocidade do vento.

Palavras-chave: modelo de temperatura; Reservatório Rio Verde; estratificação.

\section{Abstract}

Changes in the water temperature and in the thermal stratification dynamics can be responsible for negative effects on physical, chemical and biological processes of reservoirs. The temperature variation affects the water density and, consequently, alters the transport processes. A one-dimensional model of thermal evolution is used to simulate the temperature of Rio Verde reservoir, located in the Curitiba metropolitan area, Paraná state, Brazil. The finite difference method was employed for the numerical solution of the equation. The model was calibrated and validated by means of the data from two different months: July 2009 and March 2010. The results show a good agreement between simulated and observed values. These results still allow evaluating the relationships between the depth of the thermocline with solar radiation and wind speed.

Keywords: temperature model; Rio Verde Reservoir; stratification.

\section{Introdução}

A construção de reservatórios para armazenamento de água e abastecimento humano ou industrial começou no Brasil a partir do início do século passado. A criação de reservatórios pode gerar impactos ambientais negativos, como a modificação das condições de escoamento, por meio da transformação de um ambiente lótico em lêntico. Essas modificações alteram o regime térmico dos fluxos, além de introduzir novas condições químicas e biológicas ao meio. Em reservatórios, a escala espacial dos fenômenos relacionados à qualidade da água se dá preferencialmente na direção vertical, considerando-se que a dominância dos processos de transporte nesses sistemas acontece ao longo da profundidade, com poucas variações das direções transversais e longitudinais. Outra característica importante nos reservatórios, relacionada à maior profundidade e à baixa velocidade longitudinal, é a estratificação térmica e química, principalmente na região próxima a barragem

A radiação solar que atinge a superfície do lago é transformada em calor, principalmente próximo à superfície. Esse processo de transferência ocorre de forma lenta, gerando camadas de diferentes temperaturas e, consequentemente, de diferentes densidades. O vento é o principal forçante capaz de misturar tais camadas. Se a energia 
gerada pelo vento não é suficiente para promover a mistura, a coluna d'água apresenta-se estratificada por longos períodos. Sendo assim, a formação da estratificação pode ser bastante resistente à mistura, formando três camadas distintas: epilímnio, hipolímnio e metalímnio. A camada de mistura pode ser restrita ao epilímnio ou avançar até o hipolímnio, fazendo com que a circulação vertical, principalmente provocada pelo vento, ocorra ao longo de toda a coluna de água (ANDREOLI et al., 2011). No metalímnio, encontra-se a termoclina, que corresponde ao plano que passa no ponto de inflexão do perfil térmico e cuja formação ocorre por interações entre a turbulência gerada pelo vento e o empuxo devido ao gradiente de densidade; esse processo de formação é instável mesmo quando as condições ambientais são estáveis.

Em um dos primeiros estudos, Ragotzkie (1978) desenvolveu um modelo capaz de predizer a profundidade da termoclina em função do fetch, a máxima distância na superfície do reservatório em que o vento pode atuar, sem obstáculos. Gorham e Boyce (1989) mostraram que a profundidade da camada de mistura é influenciada por fatores geométricos, como área superficial e profundidade máxima do reservatório. Sperling et al. (2004) mostram a influência da radiação solar incidente sobre a formação dos perfis de temperatura e a estrutura térmica de um lago em formação, destacando a variação temporal da estabilidade térmica da coluna d'água ao longo de dois anos de medição.

Com o objetivo de prever o perfil térmico, vários autores têm desenvolvido modelos matemáticos que descrevem o comportamento da estratificação em lagos e reservatórios (LOSORDO \& PIEDRAHITA, 1991; ANTONOPOULOS \& GIANNIOU, 2003; MOMMI \& ITO, 2008). No Brasil, Pereira e Tassin (1995) desenvolveram e aplicaram um modelo para descrever o regime térmico de um reservatório tropical na região norte, obtendo bons resultados para valores de temperatura em períodos de estratificação e de mistura completa da coluna d'água. A principal vantagem da aplicação desses modelos reside na possibilidade do estudo de cenários diferentes, de forma rápida e com baixo custo. Devido à grande profundidade e baixa velocidade longitudinal, em modelos para reservatórios assume-se que os gradientes laterais podem ser desprezados quando comparados aos verticais (HENDERSON-SELLERS, 1984).

Este trabalho mostra o desenvolvimento de um modelo unidimensional de transferência de calor, com o objetivo de descrever o comportamento térmico do reservatório do Rio Verde, localizado na Região Metropolitana de Curitiba (RMC). Trata-se de um lago monomítico quente, que apresenta uma circulação completa por ano e estratificação térmica nos meses mais quentes. O modelo desenvolvido é baseado na equação de transferência de calor e tem produzido resultados satisfatórios em diversos trabalhos (ANTONOPOULOS \& GIANNIOU, 2003; CANCELLI, 2006).

Para o desenvolvimento e calibração do modelo, utilizou-se o reservatório do Rio Verde, pela disponibilidade de dados necessários para o desenvolvimento da modelagem. No reservatório, parâmetros meteorológicos e valores de temperatura da água foram medidos, a cada 15 minutos, desde 2009. Deste modo, o reservatório do Rio Verde foi escolhido para este estudo, sendo possível calibrar e validar o modelo proposto e ainda verificar as correlações entre a profundidade da termoclima, radiação solar incidente e vento.

\section{Metodologia}

O reservatório do Rio Verde, localizado na longitude 49³1'W e na latitude $25^{\circ} 31^{\prime}$, possui área de 5.971.731,0 $\mathrm{m}^{2}$, profundidade média de $5,6 \mathrm{~m}$, volume de $25.543 .732 \mathrm{~m}^{3}$ na cota do vertedouro e é orientado na direção Nordeste-Sudoeste. Segundo Andreoli et al. (2011), o reservatório pode ser considerado mesotrófico, com uma concentração média de $6,9 \mu \mathrm{g} / \mathrm{L}$ de clorofila-a e tempo de residência médio de 218 dias. De acordo com Bowie et al. (1985), é comum o uso de modelos unidimensionais em sistemas com longo tempo de residência e com estratificação vertical, características presentes no reservatório em estudo.

A bacia de contribuição abrange os municípios de Campo Magro, Campo Largo e Araucária, municípios da RMC. Concluído em 1976, o reservatório foi construído com o objetivo de abastecer a Refinaria Presidente Vargas (REPAR), localizada no município de Araucária (ANDREOLI et al., 2011). A bacia do Rio Verde integra a bacia do Alto Iguaçu e situa-se na zona climática classificada como temperada úmida, com verões moderados e sem estação seca bem definida. O clima é do tipo subtropical, com verões úmidos e invernos brandos, sendo comum a ocorrência de geadas. Os ventos da direção leste são predominantes, com intensidades mais frequentes entre 2,0 e 4,0 m.s. $(\sim 38 \%)$ e entre 4,0 e 6,0 m.s.1 ( 26\%). As velocidades médias e máximas estão entre 2,8 e 10,3 m.s. $\mathrm{s}^{-1}$, respectivamente (ANDREOLI et al., 2011).

O reservatório Rio Verde vem sendo monitorado desde 1987 pelo Instituto Ambiental do Paraná (IAP) e apresenta as seguintes características limnológicas básicas: distribuição de oxigênio dissolvido que acompanha o perfil térmico, com decréscimo na concentração de oxigênio a partir da zona eufótica e ocorrência de anoxia nas camadas de fundo, especialmente nos meses de primavera e verão; concentração média de fósforo total de $0,015 \mathrm{mg} \cdot \mathrm{L}^{-1}$; relação nitrogênio/fósforo indicando que o fósforo é o nutriente limitante à produção primária do fitoplâncton. Durante o período de monitoramento, apenas uma floração de cianobactérias foi observada, em abril de 2005.

As variáveis meteorológicas no reservatório do Rio Verde foram medidas a partir de abril de 2009, coletadas na estação meteorológica instalada próxima ao reservatório, na latitude 25³1'36,83"S e longitude 49³1'39,07'W (Figura 1), com a capacidade de medir direção e velocidade do vento, temperatura do ar, radiação incidente e umidade. Acoplados ao datalogger da estação, foram instalados quatro sensores de temperatura, a 2,0 (T1), 3,5 (T2), 5,5 (T3) e 7,0 (T4) $\mathrm{m}$ abaixo da superfície livre, para medição da temperatura da água, a cada 15 minutos, utilizando-se a estrutura da captação de 
água existente no local. Os sensores da estação meteorológica estão instalados a, aproximadamente, $10 \mathrm{~m}$ da superfície livre.

No ponto de monitoramento R4 (Figura 1), localizado na região mais profunda do reservatório, próximo à barragem, foram feitas medições de perfis de temperatura em dois períodos: entre julho de 2008 e julho de 2009 e entre março e dezembro de 2010 (Figura2). A estratificação térmica observada nesse período é evidente, mas apresenta-se variável durante o ano. Observa-se que o reservatório do Rio Verde não apresenta estratificação significativa entre os meses de maio e agosto e que, a partir de setembro, ocorre uma tendência maior à estratificação, provocada por um aquecimento da camada superior. Verifica-se que, durante o período de observação entre julho de 2008 e julho de 2009, ocorrem três fases distintas: de maio (outono) até agosto (inverno), a coluna d'água está bem misturada. Na segunda fase, que corresponde ao período entre setembro (inverno) e novembro (primavera), o lago começa a estratificar.
A terceira fase corresponde ao período entre dezembro (verão) e abril (outono); neste período, ocorre uma forte estratificação. O mesmo comportamento pode ser confirmado no segundo período, entre março e dezembro de 2010. Observa-se que, em março de 2010, a coluna d'água apresenta-se estratificada, mas, a partir de abril, apresenta-se misturada, mantendo esse comportamento até agosto, quando volta a sofrer estratificação. A análise dos dados mostra que o reservatório do Rio Verde apresenta uma circulação que abrange toda a coluna de água, ou seja, é um ambiente holomítico e possui temperatura da água sempre superior a $4^{\circ} \mathrm{C}$, confirmando a classificação de monomítico quente.

Considerando-se as fases descritas anteriormente e a disponibilidade de dados meteorológicos, foram escolhidos dois períodos para a simulação: julho de 2009, para caracterizar a época em que a coluna d'água está bem misturada, e março de 2010, período em que a coluna d’água apresenta-se estratificada. A Figura 3 mostra os valores de radiação solar

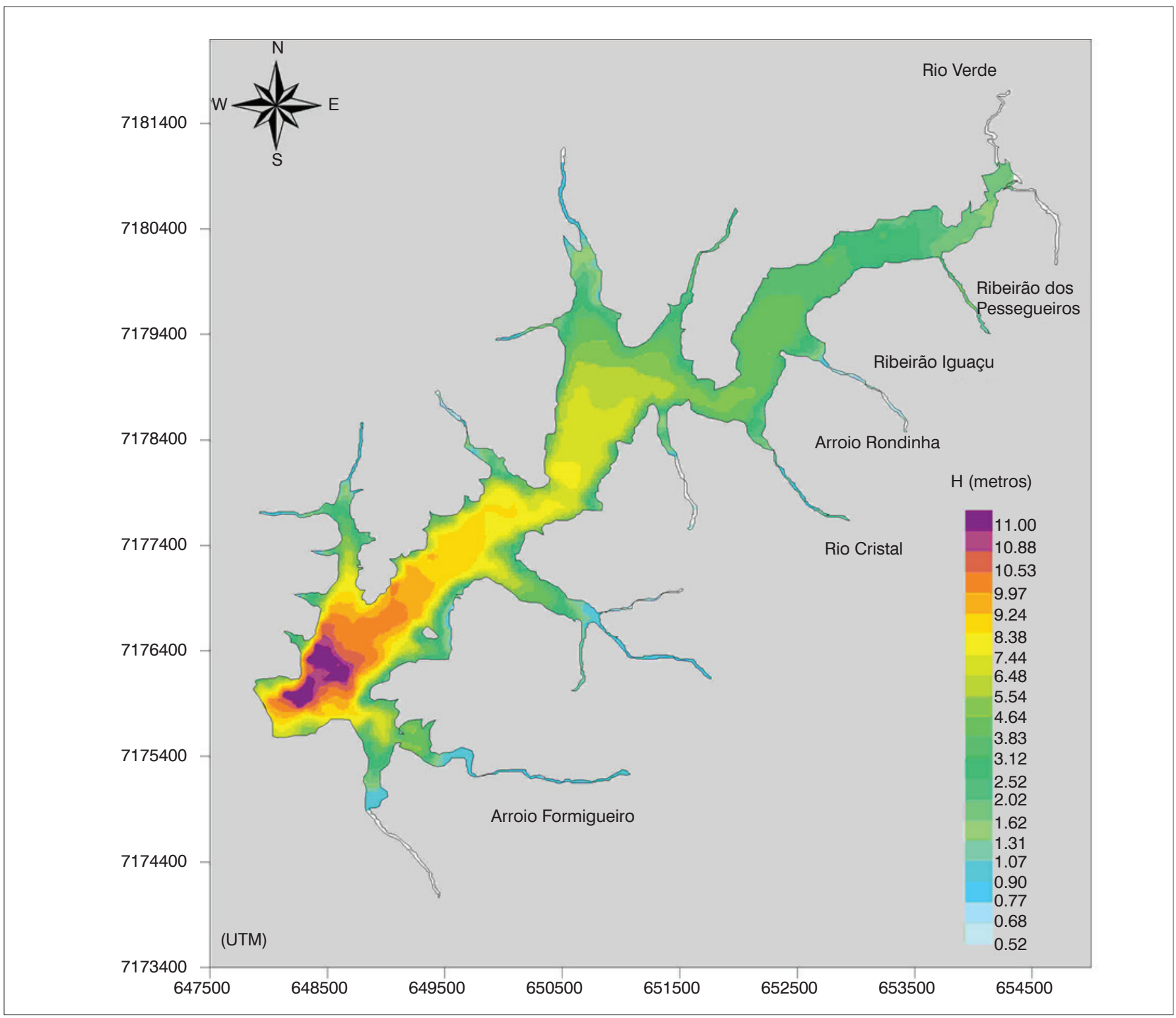

Figura 1 - Visualização da topografia de fundo e localização da estação meteorológica e do ponto de monitoramento R4 no reservatório do Rio Verde. 
incidente, temperatura do ar, umidade e velocidade do vento, usados pelo modelo de temperatura, obtidos na estação meteorológica no mês de julho de 2009. A Figura 4 mostra os mesmos valores no mês de março de 2010.

Os dados medidos de temperatura da água indicam a presença de uma estratificação em escala diária e a dependência dessas flutuações com as condições meteorológicas. Tomando o período entre 3 e 5 de julho de 2009 (Figura 5), observa-se claramente que os picos de radiação ocorrem próximos das 17h00, quando há alta irradiância solar, gerando estratificação. Há períodos em que a flutuação térmica da água é pequena, principalmente devido à baixa irradiância solar, provocando diferenças pequenas entre a temperatura da água nas quatro profundidades. Segundo Esteves (1998), o máximo da estratificação térmica ocorre por volta de 16h00-17h00, o que foi observado no reservatório do Rio Verde. Percebe-se também que,
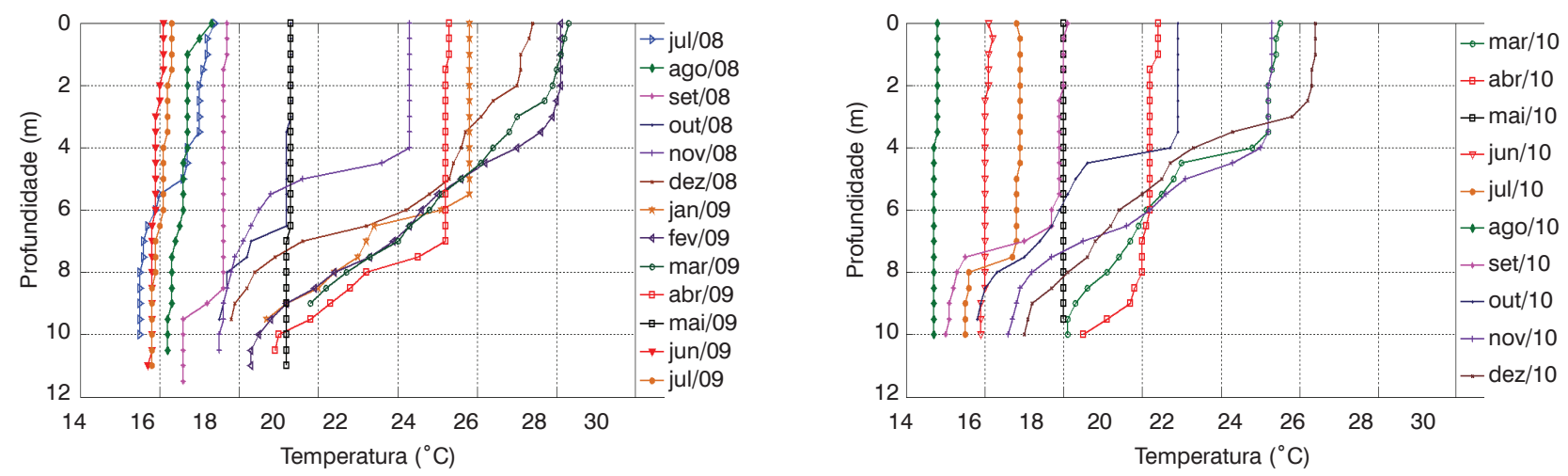

Figura 2 - Perfis de temperatura $\left({ }^{\circ} \mathrm{C}\right)$ medidos no reservatório do Rio Verde, entre julho de 2008 e julho de 2009 , e entre março e dezembro de 2010 , no ponto de monitoramento R4.

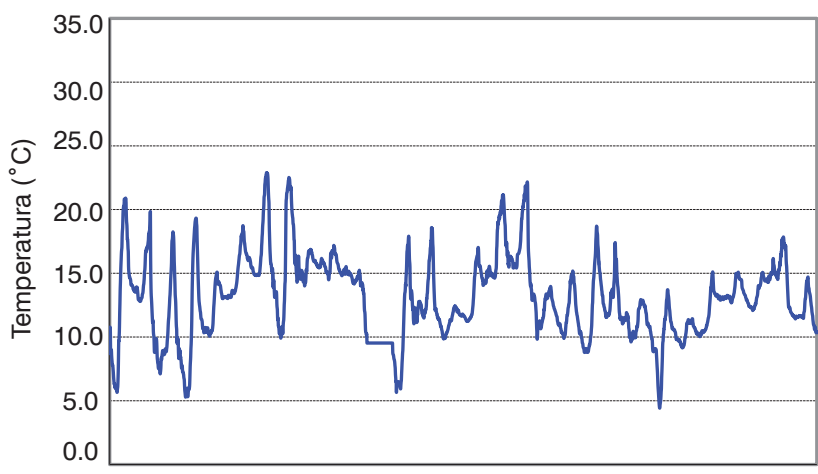

1/7/09 0:00 6/7/09 0:00 11/7/09 0:00 16/7/09 0:00 21/7/09 0:00 26/7/09 0:00 31/7/09 0:00

Data

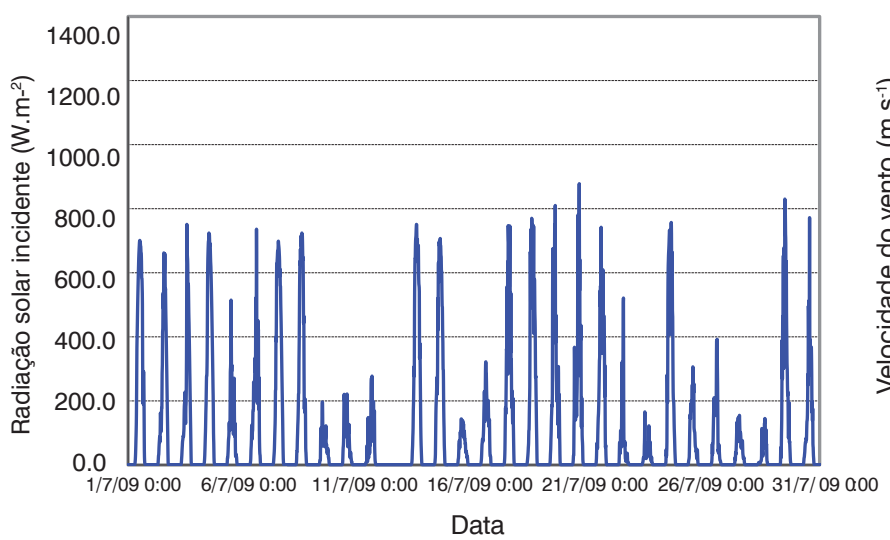

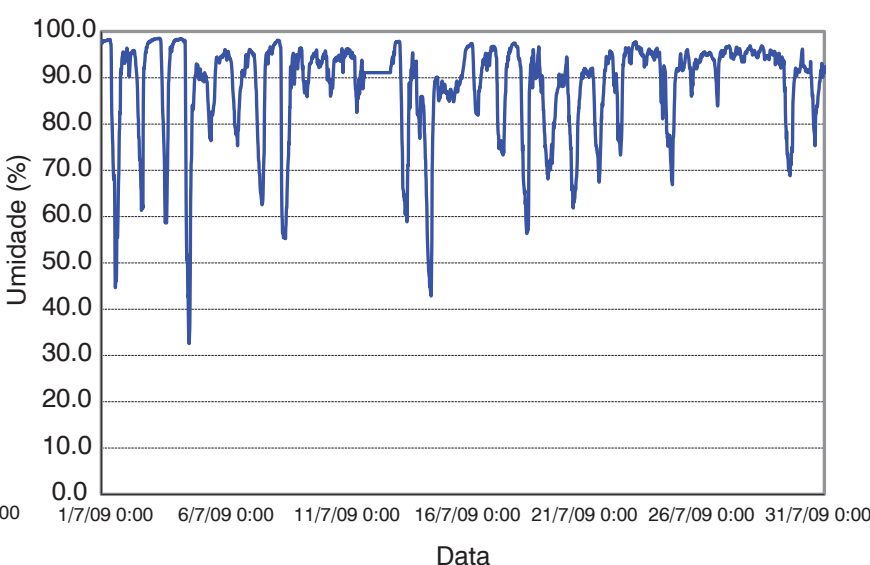

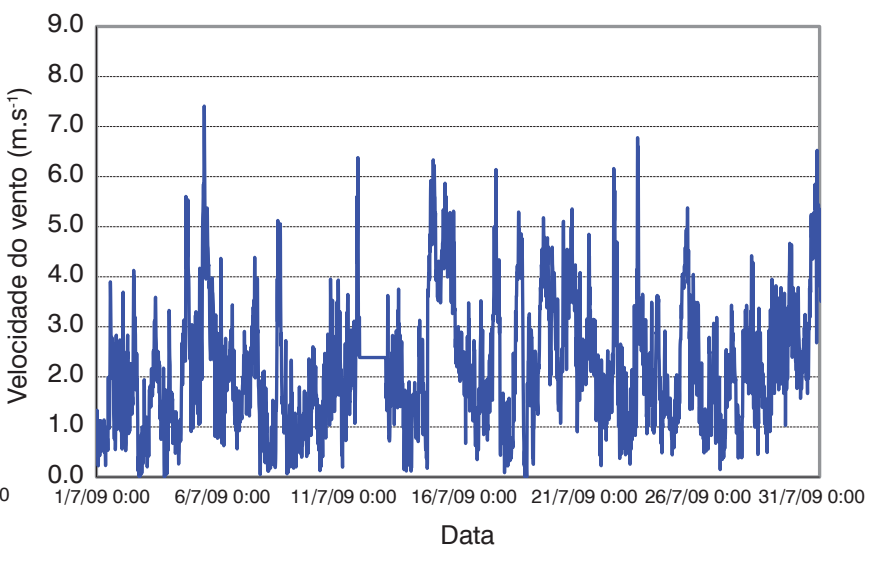

Figura 3 - Valores de temperatura, radiação solar incidente, umidade e velocidade do vento no reservatório do Rio Verde, entre $1^{\circ}$ e 31 de julho 2009. 


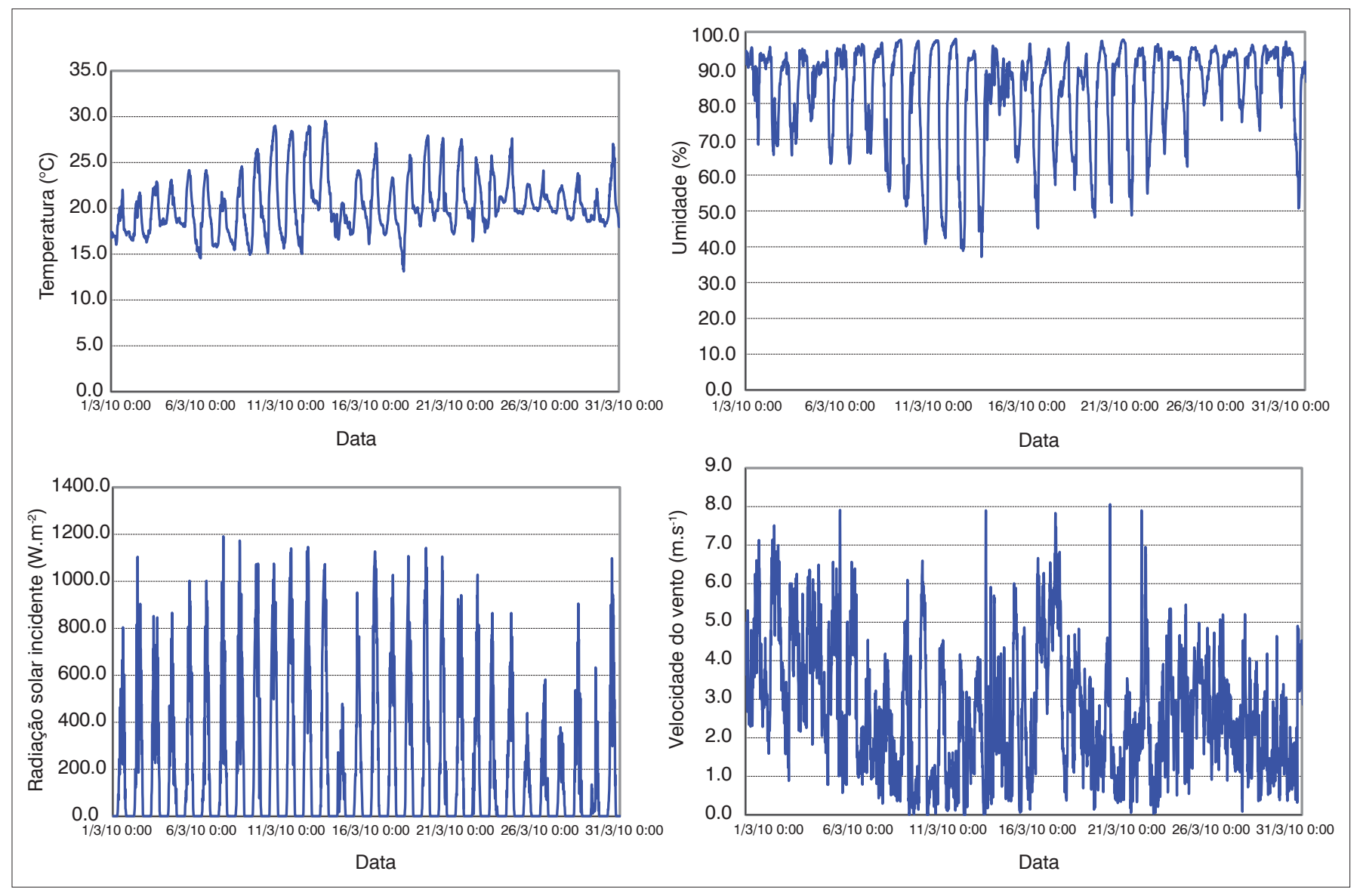

Figura 4 - Valores de temperatura, radiação solar incidente, umidade e velocidade do vento no reservatório do Rio Verde entre $1^{0}$ e 31 de março de 2010.

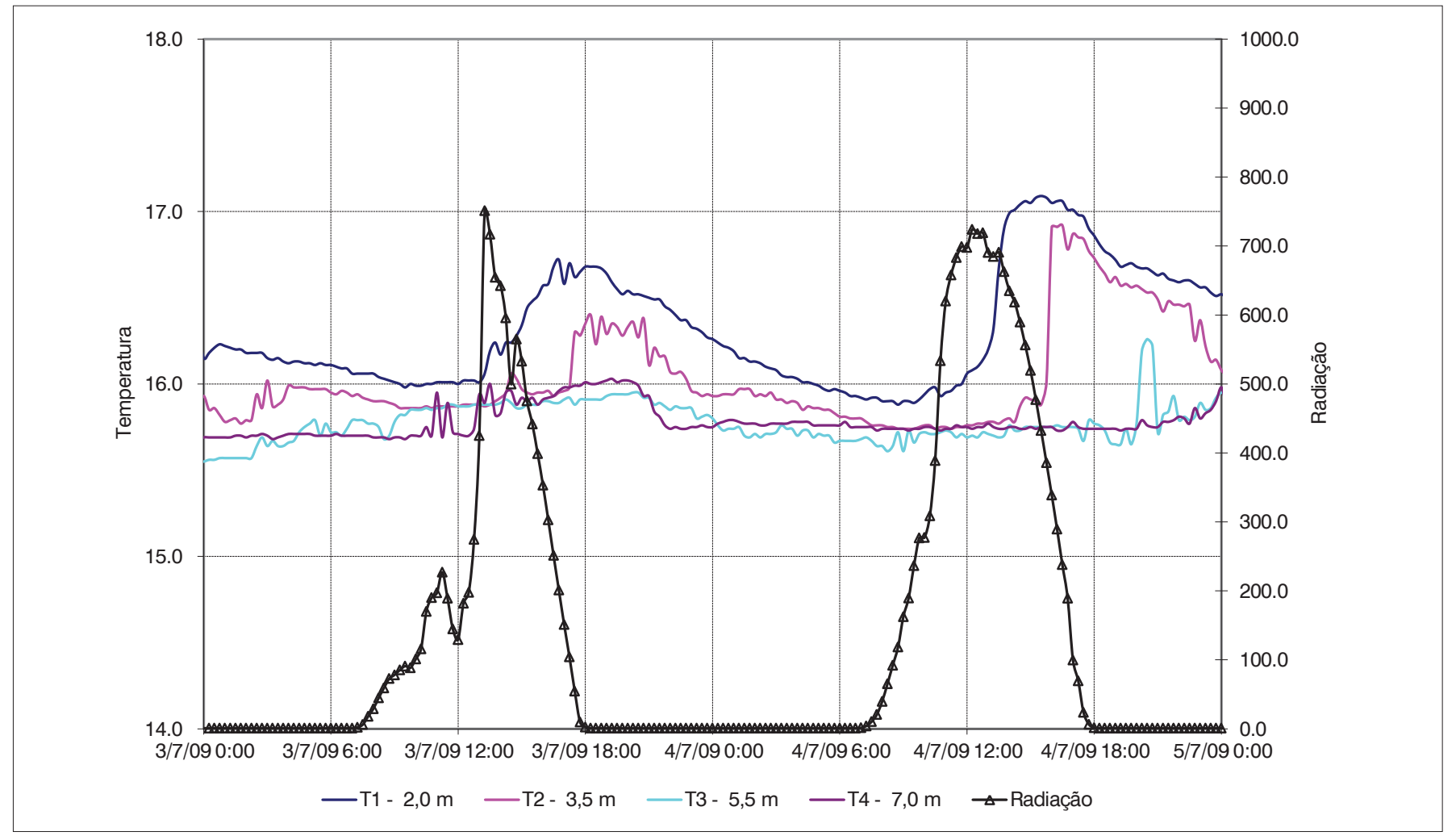

Figura 5 - Valores de temperatura $\left({ }^{\circ} \mathrm{C}\right)$ e radiação solar incidente $\left(\right.$ W. $\left.\mathrm{m}^{-2}\right)$ no reservatório do Rio Verde entre 3 e 5 de julho de 2009. 
durante a noite, ocorre uma tendência de mistura das camadas, ou desestratificação, principalmente pela perda de calor das camadas superiores. É correto afirmar também que, nas duas camadas superiores, T1 e T2, as temperaturas da água respondem às flutuações da radiação solar na escala diária, considerando-se que a maior parte da energia solar é absorvida nas camadas superiores; no entanto, na terceira camada (T3), as variações de temperaturas encontram-se defasadas em relação à radiação, ou seja, somente ocorre variação quando, no dia anterior, a irradiância solar é alta. Durante o verão, os valores de temperatura nas quatro camadas apresentam comportamento bastante distinto do observado no inverno e na primavera. Observa-se que, durante todo o período, as variações de temperatura na coluna d'água são pequenas; as camadas mais profundas não apresentam variação nos valores de temperatura, mesmo em situação de baixa irradiância solar. Em relação à estratificação diária, o reservatório do Rio Verde estratifica durante o dia e desestratifica durante noite apenas durante o inverno e início da primavera; no verão, esse comportamento não foi verificado.

Neste trabalho, as simulações de temperatura do reservatório foram realizadas mediante um modelo unidimensional de transferência de calor, dado por (Equação 1; HENDERSON-SELLERS, 1984):

$A \frac{\partial T}{\partial t}=\frac{\partial}{\partial z}\left(A K_{z} \frac{\partial T}{\partial z}\right)-\frac{1}{\rho c_{p}} \frac{\partial A q}{\partial z}$

Onde,

$\mathrm{A}(\mathrm{z})=$ área horizontal em função da profundidade $\left(\mathrm{m}^{2}\right)$;

$\mathrm{T}(\mathrm{z}, \mathrm{t})=$ temperatura da água $\left({ }^{\circ} \mathrm{C}\right)$;

$\mathrm{t}=$ tempo;

$\mathrm{z}=$ profundidade;

$\mathrm{K}_{\mathrm{z}}=$ coeficiente de difusão $\left(\mathrm{m}^{2} \cdot\right.$ dia $\left.^{-1}\right)$, dado pela soma da difusão molecular, $\mathrm{D}_{0}$, com a difusão turbulenta, $\mathrm{E}(\mathrm{z}, \mathrm{t})$;

$\mathrm{q}(\mathrm{z})=$ distribuição interna de calor dentro da coluna de água devido à absorção da radiação solar (cal.m-2 dia $\left.{ }^{-1}\right)$;

$\rho=$ massa específica da água $\left(\mathrm{kg} \cdot \mathrm{m}^{-3}\right)$;

$\mathrm{c}_{\mathrm{p}}=$ calor específico da água $\left(\mathrm{cal}^{\circ}{ }^{\circ} \mathrm{C}^{-1} \mathrm{~g}^{-1}\right)$.

A condição de contorno da superfície $(z=0,0)$ descreve as transferências de calor entre o reservatório e a atmosfera (Equação 2):

$q_{n}=-\left.\rho c_{p} K_{z} \frac{\partial T}{\partial z}\right|_{z=0}$

Onde:

$\mathrm{q}_{\mathrm{n}}=$ fluxo de calor na superfície linearizado.

No fundo, a condição de contorno consiste na ausência de fluxo de calor (Equação 3):

$\left.\frac{\partial \mathrm{T}}{\partial z}\right|_{z=\mathrm{H}}=0$
Onde,

$\mathrm{H}=$ profundidade no fundo do reservatório.

O termo $q_{n}$ representa o fluxo de calor na superfície linearizado, que faz uso da temperatura de equilíbrio e do coeficiente de troca de calor na superfície (Equação 4; THOMANN \& MULLER, 1987):

$\mathrm{q}_{\mathrm{n}}=-\mathrm{K}\left(\mathrm{T}_{\mathrm{s}}-\mathrm{T}_{\mathrm{e}}\right)$

Onde:

$\mathrm{K}=$ coeficiente de troca de calor na superfície $\left(\mathrm{W}^{\circ} \mathrm{C}^{-1} \mathrm{~m}^{-2}\right)$;

$\mathrm{T}_{\mathrm{s}}=$ temperatura na superfície $\left({ }^{\circ} \mathrm{C}\right)$;

$\mathrm{T}_{\mathrm{e}}=$ temperatura de equilíbrio.

A temperatura de equilíbrio é uma temperatura fictícia, na qual não haveria troca de calor na superfície do reservatório $\left(q_{n}=0\right)$, podendo ser avaliada por (Equação 5; THOMANN \& MULLER, 1987):

$\mathrm{T}_{\mathrm{e}}=\mathrm{T}_{\mathrm{d}}+\mathrm{q}_{\mathrm{sn}} / \mathrm{K}$

Onde,

$\mathrm{T}_{\mathrm{d}}=$ temperatura do ponto de orvalho do $\operatorname{ar}\left({ }^{\circ} \mathrm{C}\right)$;

$\mathrm{q}_{\mathrm{sn}}=$ radiação solar líquida de ondas curtas $\left(\mathrm{W} \cdot \mathrm{m}^{-2}\right)$.

O coeficiente de troca de calor na superfície é avaliado pela expressão (Equação 6; ANTONOPOULOS \& GIANNIOU, 2003):

$K=4,5+0,05 T_{s}+\delta f(u)+0,47 f(u)$

Onde (Equações 7 a 9),

$$
\begin{aligned}
& \mathrm{f}(\mathrm{u})=9,2+0,46 \mathrm{u}^{2} \\
& \delta=0,35+0,015 \mathrm{~T}_{\mathrm{m}}+0,0012 \mathrm{~T}_{\mathrm{m}}^{2} \\
& \mathrm{~T}_{\mathrm{m}}=\left(\mathrm{T}_{\mathrm{s}}+\mathrm{T}_{\mathrm{d}}\right) / 2
\end{aligned}
$$

O parâmetro u nas expressões anteriores representa a velocidade do vento medida a $2 \mathrm{~m}$. A distribuição da radiação solar em função da profundidade pode ser descrita como (Equação 10):

$q(z)=(1-\beta) q_{s n} \exp (-\eta z)$

Onde,

$\eta=$ coeficiente de extinção (ou absorção) de radiação solar na água $\left(\mathrm{m}^{-1}\right)$; $\beta=$ fração de $q_{\mathrm{sn}}$ absorvida na superfície; $\mathrm{q}_{\mathrm{sn}}=$ radiação solar líquida de ondas curtas $\left(\mathrm{cal} \cdot \mathrm{m}^{-2} \mathrm{dia}^{-1}\right)$.

A fração de radiação absorvida na superfície $(\beta)$ pode ser obtida pela Lei de Beer, que avalia a absorção exponencial. Um valor constante de $\beta$ sugerido é de 0,4 a 0,5. Entretanto, esse valor também 
pode variar com a turbidez da água (HERDERSON-SELLERS, 1984). O coeficiente de extinção vertical $(\eta)$ corresponde à fração de luz absorvida por metro de coluna d'água. Quanto maior o valor de $\eta$, menor é a transmissão da luz e, consequentemente, menor a transparência da massa líquida. Os maiores valores médios de $\eta$ são encontrados em lagos túrbidos, com alta concentração de compostos em suspensão. Pode-se, ainda, estimar $\eta$ em relação à profundidade do disco de Secchi (d): $\eta=1,7 / d$. No reservatório do Rio Verde, a profundidade de dé 1,55 m em julho de 2009 e 1,25 m em março de 2010.

Uma das dificuldades na aplicação da Equação 1 é estimar o coeficiente de difusão turbulenta. Este pode ser baseado em valores de estudos similares ou em formulações empíricas e tem fundamental importância nos cálculos dos perfis verticais de temperatura. O coeficiente de difusão turbulenta pode ser calculado como (Equações 11 a 13):

$$
\begin{aligned}
& E=E_{0} f \\
& f=(1+\sigma R i)^{-n} \\
& E_{0}=c_{2} w^{*}
\end{aligned}
$$

Onde,

$E=$ coeficiente de difusão turbulenta;

$\mathrm{E}_{0}=$ coeficiente de difusão turbulenta em meio neutro $\left(\mathrm{m}^{2} \cdot \mathrm{s}^{-1}\right)$,

$c_{2}, \sigma$ e $n=$ constantes;

$\mathrm{W}^{*}=$ velocidade de atrito do vento $\left(\mathrm{m} . \mathrm{s}^{-1}\right)$;

Ri=número de Richardson

A velocidade de atrito $\mathrm{w}^{*}$ é obtida pela expressão (Equação 14; ANTONOPOULOS \& GIANNIOU, 2003):

$\mathrm{w}^{*}=1,2 \times 10^{-3} \mathrm{u}_{10}$

Onde,

$\mathrm{u}_{10}=$ velocidade do vento medida a $10 \mathrm{~m}\left(\mathrm{~m} \cdot \mathrm{s}^{-1}\right)$.

O número de Richardson descreve a estabilidade da coluna, e representa uma estimativa de ocorrência da mistura entre as camadas estratificadas.

O valor de Ri é dado pela razão entre as forças de empuxo e a energia para mistura causada pelo cisalhamento do vento, sendo representado por (Equação 15; HENDERSON-SELLERS, 1984):

$\mathrm{Ri}=-\frac{\mathrm{g} \Delta \rho \mathrm{h}}{\rho w^{* 2}}$

Onde,

$\mathrm{g}=$ aceleração da gravidade $\left(\mathrm{m} \cdot \mathrm{s}^{-2}\right)$;

$\Delta \rho=$ diferença de densidade entre o epilímnio e o hipolímnio $\left(\mathrm{kg} . \mathrm{m}^{-3}\right)$; $\mathrm{h}=$ profundidade de formação da termoclina, que pode ser estimada pela expressão (Equação 16; GORHAM \& BOYCE, 1989): $h=2,0\left(\frac{\tau}{g \Delta \rho}\right)^{1 / 2} L^{1 / 2}$

Onde,

$\tau=$ tensão superficial gerada pelo vento $\left(\mathrm{kg} \cdot \mathrm{m}^{-1} \cdot \mathrm{s}^{-2}\right)$;

$\mathrm{L}=$ raiz quadrada da área superficial do reservatório (m).

Neste trabalho, na camada superior de fluido, definida a partir da profundidade da termoclina, o coeficiente de difusão turbulenta é definido como constante; na camada abaixo, o coeficiente de difusão turbulenta passa a ser dado pela Equação 11, ou seja, variando com o número de Richardson. A variação da densidade foi calculada como (Equação 17; UNESCO, 1981):

$\rho_{0}=999,842594+6,793952 \times 10^{-2} \mathrm{~T}-9,095290 \times 10^{-3} \mathrm{~T}^{2}$

$+1,001685 \times 10^{-4} \mathrm{~T}^{3}-1,120083 \times 10^{-6} \mathrm{~T}^{4}+6,536332 \times 10^{-9} \mathrm{~T}^{5}$

Em geral, não é possível desenvolver uma solução analítica para a simulação da temperatura da água, devido à não lineariedade de alguns termos da expressão de fluxo de calor na interface ar-água. Deste modo, neste trabalho, adotou-se uma solução numérica para a resolução da Equação 1, com o uso de um método de diferenças finitas implícito: a derivada temporal é discretizada pelo método da fatoração implícita (CUNHA et al., 2006), e as derivadas espaciais são discretizadas por um esquema de diferenças centrais. A equação discretizada resulta em um sistema de equações lineares, resolvido pelo método direto do Algoritmo de Thomas ou TDMA.

\section{Resultados e discussão}

O modelo unidimensional de transferência de calor foi aplicado para o reservatório do Rio Verde, em dois períodos: julho de 2009 e março de 2010. O reservatório foi dividido em camadas com $\Delta z=0,5 \mathrm{~m}$, sendo que a área horizontal do reservatório foi considerada constante ao longo da profundidade. Os valores obtidos pelo modelo foram comparados com os valores de temperatura observados no reservatório (Figuras 6 e 7). Os parâmetros usados nas simulações nos dois períodos são mostrados na Tabela 1. Os valores foram obtidos a partir de dados da literatura (ANTONOPOULOS $\&$ GIANNIOU, 2003; CANCELLI, 2006) e ajustados para melhor representar os resultados. O erro médio absoluto, erro médio quadrático e a raiz quadrada do erro médio quadrático são apresentados na Tabela 2, para as duas simulações.

Na primeira simulação, realizada em julho de 2009 (Figura 6), para a situação de pouca ou nenhuma estratificação, os resultados obtidos pelo modelo podem ser considerados satisfatórios, principalmente nos primeiros dias, para as camadas mais profundas; no entanto, nas camadas superficiais, as diferenças encontradas foram 


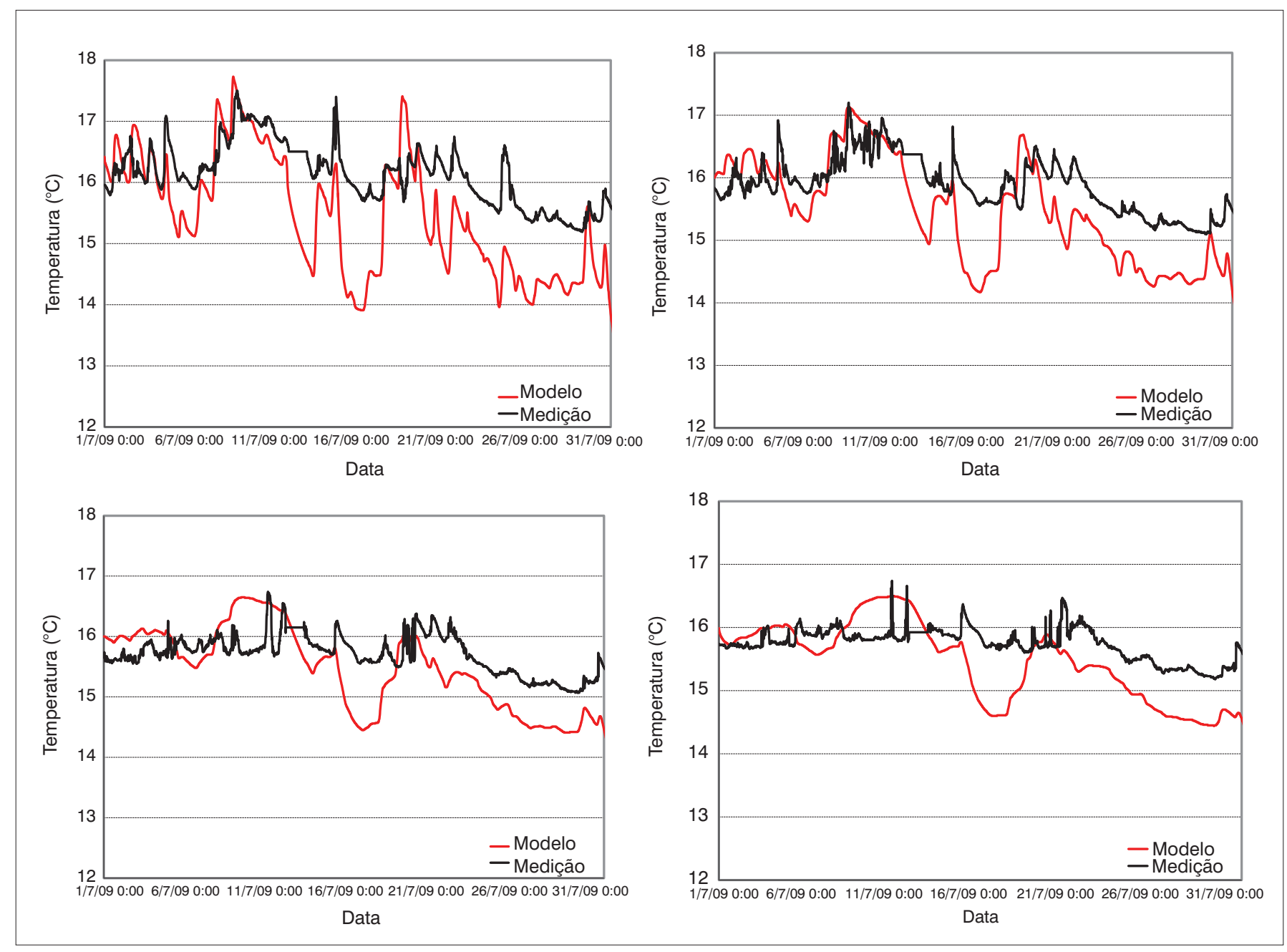

Figura 6 - Comparação entre os valores de temperatura medidos e obtidos pelo modelo, em graus Celsius, no reservatório do Rio Verde entre $1^{0}$ e 31 de julho de 2009 no ponto 2,0 $\mathrm{m}$ da superfície (acima e à esquerda), 3,5 m da superfície (abaixo e à esquerda), 5,5 m da superfície (acima e à direita), $7,0 \mathrm{~m}$ da superfície (abaixo e à direita).

Tabela 1 - Valores dos parâmetros e coeficientes usados nas duas simulações.

\begin{tabular}{lcc} 
Parâmetros e coeficientes & Julho/2009 & Março/2010 \\
$E_{0}\left(\mathrm{~m}^{2} \mathrm{~s}^{-1}\right)$ & $4,0 \times 10^{-5}$ & $4,0 \times 10^{-5}$ \\
$\eta\left(\mathrm{m}^{-1}\right)$ & 0,80 & 0,80 \\
$\beta\left(\mathrm{m}^{-1}\right)$ & 0,65 & 0,50 \\
$\mathrm{c}_{\mathrm{p}}\left(\mathrm{J}^{\circ} \mathrm{C}^{-1} \mathrm{~kg}^{-1}\right)$ & 4184 & 4184 \\
$\mathrm{C}_{2}$ & 0,15 & 0,15 \\
$\mathrm{n}$ & 1,0 & 1,0 \\
$\sigma$ & 1,0 & 1,0 \\
\hline
\end{tabular}

$E_{0}$ : coeficiente de difusão turbulenta em meio neutro; $\eta$ : coeficiente de extinção (ou absorção) de radiação solar na água; $\beta$ : fração de radiação solar líquida de ondas curtas absorvida na superfície; $c_{p}$ : calor específico da água; $c_{2} n$ e $\sigma$ : constantes.

significativas, mesmo com o modelo descrevendo as variações diárias observadas. Essas diferenças podem ser creditadas aos coeficientes de extinção de radiação solar na água e de extinção vertical, que permanecem constantes durante a simulação, mas que podem variar ao longo do tempo. No final do período, pode ter acontecido uma mudança na capacidade de absorver e transmitir calor, que não foi bem representada pelo modelo.
A Figura 7 mostra a comparação dos valores de temperatura medidos e obtidos pelo modelo para o mês de março de 2010, quando a coluna d'água apresenta-se estratificada. Novamente, nos primeiros dias de simulação, os resultados obtidos pelo modelo são satisfatórios; apenas no fundo, o modelo não foi capaz de descrever os valores medidos. Tem-se nesse mês a mesma questão das variações temporais dos parâmetros relatada na simulação anterior. No entanto, deve-se considerar que, nesta simulação, ocorre a correção do coeficiente de difusão turbulenta pela presença da estratificação, que pode explicar as diferenças nas camadas mais profundas.

Observando-se os erros obtidos nas simulações, para julho de 2009 , sem estratificação, os maiores valores são $0,85^{\circ} \mathrm{C}$ para a camada mais próxima da superfície. No mês de março de 2010, com estratificação, os erros são um pouco maiores, sendo que a camada no fundo é a que apresentou o resultado menos satisfatório. Em um estudo semelhante, Antonopoulos \& Gianniou (2003) encontraram valores da raiz quadrada do erro médio quadrático entre 0,42 e $1,84^{\circ} \mathrm{C}$ e do erro médio absoluto na ordem de 0,36 a $1,44^{\circ} \mathrm{C}$. Pereira \& Tassin (1995) 

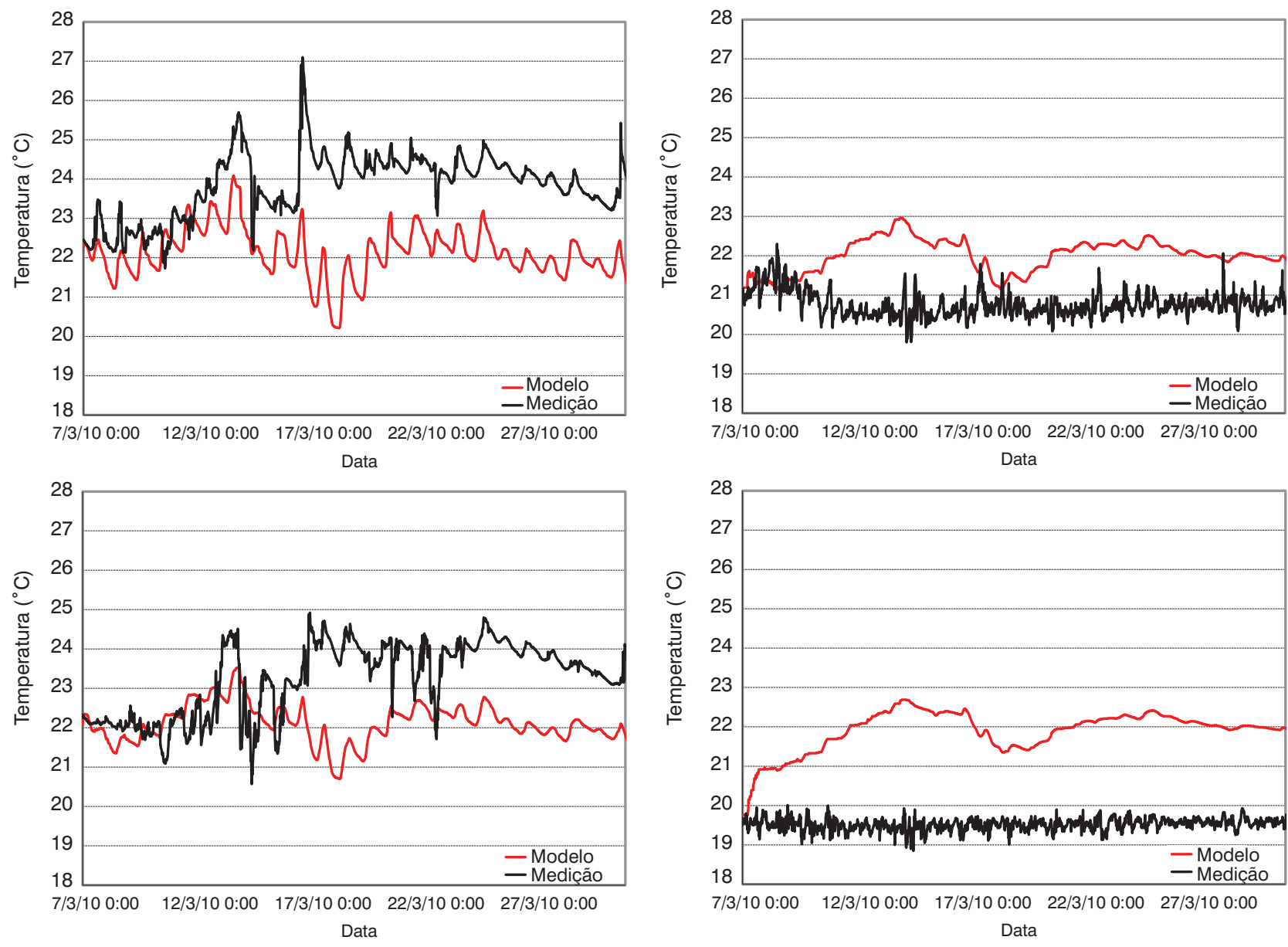

Figura 7 - Comparação entre os valores de temperatura medidos e obtidos pelo modelo, em graus Celsius, no reservatório do Rio Verde entre 7 e 31 de março de 2010 no ponto 2,0 $\mathrm{m}$ da superfície (acima e à esquerda), 3,5 m da superfície (abaixo e à esquerda), 5,5 m da superfície (acima e à direita), 7,0 m da superfície (abaixo e à direita).

Tabela 2 - Erro médio absoluto, erro médio quadrático e raiz quadrada do erro médio quadrático para as simulações em julho de 2009 e março de 2010.

\begin{tabular}{|c|c|c|c|c|c|c|c|c|}
\hline \multirow[b]{2}{*}{ Profundidade (m) } & \multicolumn{4}{|c|}{ Julho/2009 } & \multicolumn{4}{|c|}{ Março/2010 } \\
\hline & 2,0 & 3,5 & 5,5 & 7,0 & 2,0 & 3,5 & 5,5 & 7,0 \\
\hline Erro médio absoluto $\left({ }^{\circ} \mathrm{C}\right)$ & 0,85 & 0,63 & 0,54 & 0,51 & 1,77 & 1,36 & 1,27 & 2,37 \\
\hline Erro médio quadrático $(\stackrel{\circ}{\mathrm{C}})$ & 1,03 & 0,55 & 0,39 & 0,37 & 3,93 & 2,50 & 1,92 & 5,93 \\
\hline Raiz quadrada do erro médio quadrático & 1,02 & 0,74 & 0,62 & 0,61 & 1,98 & 1,58 & 1,39 & 2,43 \\
\hline
\end{tabular}

utilizaram um critério de erro análogo em suas análises e obtiveram um erro máximo diário de $1,8^{\circ} \mathrm{C}$ para uma simulação de um ano. Em outro estudo, Lam \& Schertzer (1987) encontraram um erro médio relativo entre temperaturas medidas e computadas de $5 \%$.

Outro objetivo do trabalho foi buscar correlações entre a profundidade da termoclina, calculada a partir da Equação 16, com a intensidade do vento e com a radiação solar incidente, principais forçantes responsáveis pela estratificação e pela mistura. Essas relações podem ser observadas na Figura 8. Os resultados mostram que a estratificação acontece quando ocorre a maior disponibilidade de radiação, que aquece a camada superior de água; o aquecimento das camadas mais profundas é mais lento. Esse comportamento pode ser observado nos dois períodos simulados.

Observa-se que, em geral, baixos níveis de radiação solar ocasionam mistura da coluna, ou seja, a termoclina não é formada (a profundidade permanece $12 \mathrm{~m}$ ). Esse comportamento pode ser observado principalmente em julho de 2009, quando a coluna d'água encontra-se misturada. De acordo com Fee et al. (1996), em lagos 

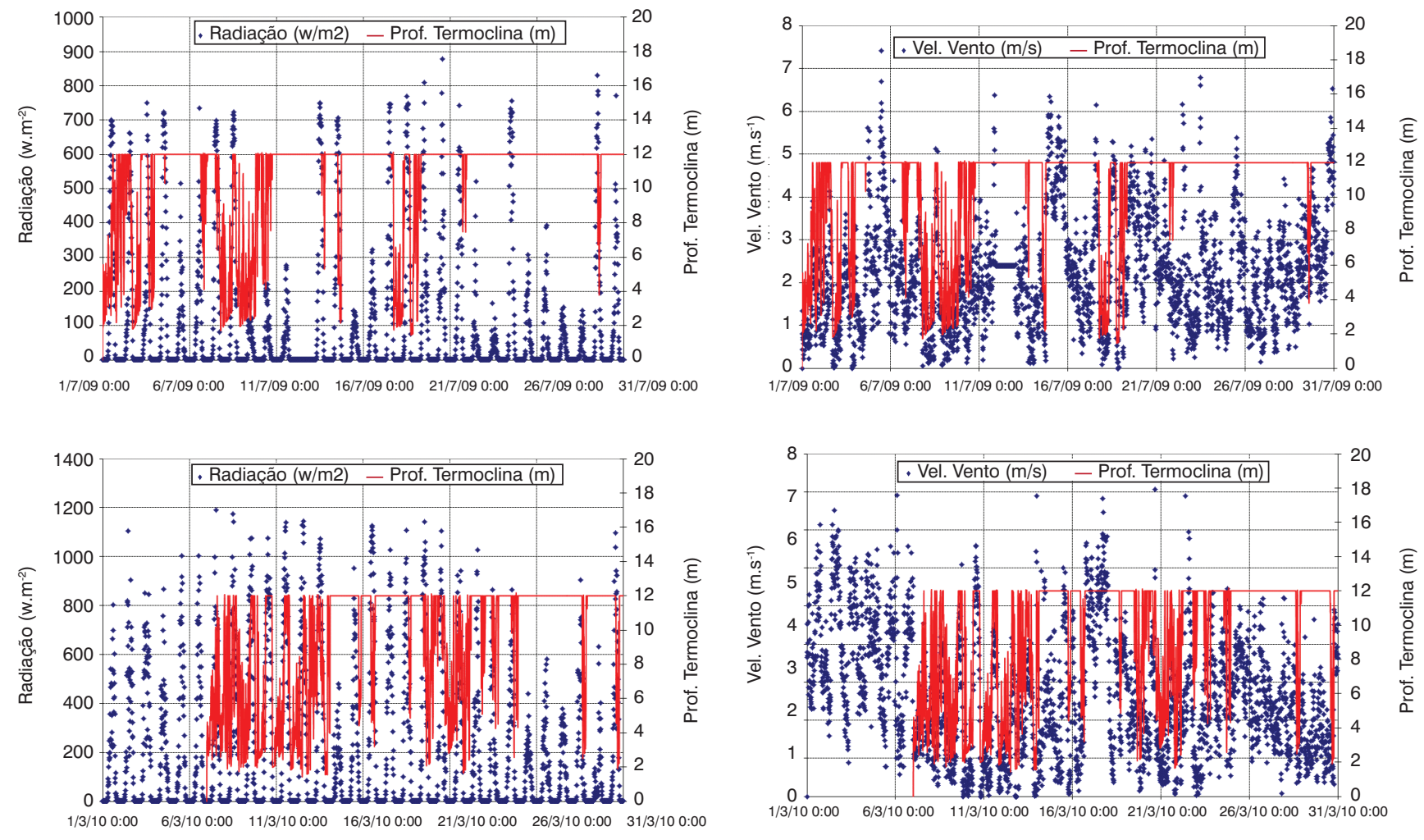

Figura 8 - Valores de radiação solar incidente, velocidade do vento e profundidade da termoclina, entre $1^{0}$ e 31 de julho de 2009 e $1^{0}$ e 31 de março de 2010.

pequenos, o aprofundamento da termoclina depende principalmente da radiação incidente, enquanto o efeito do vento é maior em lagos maiores. E, ainda, de acordo com Caprariis (1981), não é clara a existência de uma correlação significativa entre as flutuações da velocidade do vento e o perfil de temperatura. Isso foi observado nas simulações de julho de 2009 e de março de 2010, em que a velocidade do vento não apresentou correspondência significativa com a variação da profundidade da termoclina.

\section{Conclusões}

Este trabalho mostra uma simulação numérica do comportamento térmico do reservatório do Rio Verde, por meio do desenvolvimento e da aplicação de um modelo unidimensional de transferência de calor. O modelo desenvolvido mostrou-se estável, sendo capaz de estimar os valores de temperatura em várias profundidades. A validação dos resultados do modelo foi feita pela comparação com resultados medidos, considerando-se diferentes parâmetros de simulação. Os resultados mostram que os erros obtidos nas simulações são maiores no mês de março, quando ocorre estratificação, e menores em julho, quando a coluna está misturada. A ordem de grandeza dos erros observados está coerente com os valores observados na literatura. Os erros são devidos, principalmente, à pouca precisão das estimativas de fluxos de calor, calculados a partir de dados meteorológicos medidos em uma estação instalada próximo ao reservatório. Foi possível, ainda, avaliar qualitativamente a influência da radiação solar e da velocidade do vento sobre a variação da profundidade da termoclina. Observou-se que a formação da estratificação térmica no reservatório depende principalmente da radiação solar incidente e que o vento não é eficiente em misturar a coluna de água.

Os resultados obtidos indicam que a acurácia do modelo desenvolvido pode ser aprimorada, principalmente em relação ao cálculo do coeficiente de difusão turbulenta. A simulação correta do regime térmico de um reservatório é essencial para o conhecimento dos processos limnológicos, uma vez que a posição da termoclina representa uma informação importante para o estudo das condições do hipolímnio, possibilitando o conhecimento das variações da produção primária. Consequentemente, a previsão dos valores de temperatura, em lagos subtropicais, ainda é um desafio. 


\section{Referências}

ANDREOLI, C.V.; CARNEIRO, C.; GUTSEIT, K.; XAVIER, C.F. (2011) Caracterização geral da bacia. In: CUNHA, C.L.N. Eutrofização em reservatórios: gestão preventiva. Estudo interdisciplinar na bacia do Rio Verde, PR. Curitiba: UFPR. p. 39-58.

ANTONOPOULOS, V.Z \& GIANNIOU, S.K. (2003) Simulation of water temperature and dissolved oxygen distribution in Lake Vegoritis, Greece. Ecological Modelling, v. 160, n. 1-2, p. 39-53.

BOWIE, G.L.; MILLS, W.B.; PORCELLA, D.B.; CAMPBELL, C.L.; PAGENKOPF, J.R.; RUPP, G.L.; JOHNSON, K.M.; CHAN, P.W.H.; GHERINI, S.A.; CHAMBERLIN, C.E.; TETRA TECH, INC. (1985) Rates, Constants, and Kinetics Formulations in Surface Water Quality Modeling. 2 ed. U.S. Environmental Protection Agency, EPA/600/3-85/040.

CANCELLI, D.M. (2006) Um modelo para a evolução térmica de lagos profundos. Dissertação (Mestrado em Métodos Numéricos em Engenharia) - Universidade Federal do Paraná, Curitiba.

CAPRARIIS, P. (1981) A note on the development of the thermocline in temperate lakes. Ecological Modelling, v. 12, n. 4, p. 213-219.

CUNHA, C.L.N.; ROSMAN, P.C.C.; FERREIRA, A.P.; MONTEIRO, T.C.N. (2006) Hydrodynamics and Water Quality Models Applied to Sepetiba Bay. Continental Shelf Research, v. 26, p. 1940-1953.

ESTEVES, F.A. (1998) Fundamentos de Limnologia. 2 ed. Rio de Janeiro: Interciência.

FEE, E.J.; HECKY, R.E.; KASIAN,S.E.M.; CRUIKSHANK, D.R. (1996) Effects of lake size, water clarity, and climatic variability on mixing depths in Canadian Shield lakes. Limnology and Oceanography, v. 41, n. 5, p. 912-920.

GORHAM, E. \& BOYCE, F.M. (1989) Influence of lake surface area and depth upon thermal stratification and the depth of the summer thermocline. Journal Great Lakes Research, v. 15, n. 2, p. 233-245.
HENDERSON-SELLERS, B. (1984) Engineering limnology. London: Pitman Publishing

LAM, D.C.L. \& SCHERTZER, W.M. (1987) Lake Erie thermocline model results: comparison with 1976-1982 data and relation to anoxic occurrences. Journal Great Lakes Research, v. 13, n. 4, p.757-769.

LOSORDO, T.M. \& PIEDRAHITA, R.H. (1991) Modelling temperature variation and termal stratification in shallow aquaculture ponds. Ecological Modelling, v. 54, n. 3-4, p. 189-226.

MOMMI, K. \& ITO, Y. (2008) Heat budget estimates for Lake Ikeda, Japan. Journal of Hydrology, v. 361, n. 3-4, p. 362-370.

ORGANIZAÇÃO DAS NAÇÕES UNIDAS PARA A EDUCAÇÃO, A CIÊNCIA E A CULTURA (UNESCO) (1981). Tenth report of the joint panel on oceanographic tables and standards. Paris: UNESCO. (UNESCO Technical Papers in Marine Science 36).

PEREIRA, A. \& TASSIN, B. (1995) Modelagem matemática do regime térmico do reservatório do Tucuruí. Revista Brasileira de Engenharia (RBE) - Caderno de Recursos Hídricos, v. 13, n. 1, p. 57-76.

RAGOTZKIE, R.A. (1978) Heat budget of lakes. In: LERMAN, A. Lakes: Chemistry, Geology, Physics. New York: Springer-Verlag. p. 1-19.

SPERLING, E.V.; JARIDM, F.A.; GRANDCHAMP, C.A.P. (2004) Qualidade da água durante a formação de lagos profundos em cavas de mineração: estudo de caso do lago de Águas Claras MG. Engenharia Sanitária e Ambiental, v. 9, n. 3, p. 250-259.

THOMANN, R.V. \& MULLER, J.A. (1987) Principle of surface water quality modeling and control. New York: Harper Collins. 